\title{
Tissue Doppler and Speckle Tracking Echocardiography Assessment of Left Ventricular Function in Children with Cystic
} Fibrosis

\author{
Ozgur Kizilca ${ }^{1}$, Tulay Demircan ${ }^{1}$, Sakine $\mathrm{Isik}^{1}$, Nuh Yilmaz ${ }^{1}$, Mustafa Kır ${ }^{1}$, Nevin Uzuner $^{1}$, \\ and Nurettin Unal ${ }^{1}$ \\ ${ }^{1}$ Dokuz Eylül University
}

May 6, 2020

\begin{abstract}
Abstract Background: Cystic fibrosis may lead to left ventricular dysfunction. This dysfunction can be documented by methods such as tissue doppler echocardiographic imaging and two-dimensional speckle tracking echocardiography in early stage. Patients and Methods: A total of 34 patients diagnosed with cystic fibrosis (mean age and SD 9.9 \pm 4.9 years) and 37 healthy control subjects with a comparable gender and age distribution (mean age $9.8 \pm 4.3$ ) were studied. The results for the two groups were compared along with the results of published reports. Result: Control group had higher diastolic and systolic dimentions compared to the patient group in M-mode measurements of left ventricle by conventional echocardiography $(\mathrm{p}<0.05)$. There was no significant relationship between the groups in terms of the dimensions of systolic and diastolic measurements of interventricular septum and posterior wall of left ventricle, and ejection fraction. Pulmonary artery systolic pressure was significantly higher in the patient group $(\mathrm{p}<0.001)$. Myocardial performance indices of left ventricle free wall and interventricular septum were increased in the patient group compared to the control group $(\mathrm{p}<0.05)$. ). As measured by speckle tracking echocardiography, 7 segments in left ventricular myocardial longitudinal strain and 3 segments in left ventricular myocardial circumferential strain showed significant reductions in patients with cystic fibrosis compared to controls $(\mathrm{p}<0.05)$. Conclusions: Tissue doppler echocardiographic imaging and speckle tracking echocardiography may help identifying subclinical left ventricular dysfunction in cystic fibrosis patients with unremarkable conventional echocardiography. Its may be considered for the routine follow-up of cystic fibrosis patients.
\end{abstract}

\section{Introduction}

Cystic fibrosis $(\mathrm{CF})$ is an autosomal recessive hereditary disease with a chronic, progressive, multisystemic course $(1,2)$. The patients show a deficiency of the gene coding for the transmembrane conductance regulator protein (CFTR) (2,3). CF is a systemic disease causing multiple organ malfunctions (5). The classical triad of signs include suppurative chronic obstructive pulmonary disease, pancreatic insufficiency and high sweat electrolyte levels (7). Pulmonary injury developing out of chronic lung infection and inflammation is the most important morbidity and mortality factor (4). Pulmonary vasoconstriction develops following alveolar hypoxia resulting from pulmonary damage. This, in its turn, contributes to the development of cor pulmonale, its most frequent cardiac complication $(7,8)$. Pulmonary hypertension, myocardial fibrosis and chronic infection combine to cause right ventricular (RV) dysfunction. Left ventricular (LV) dysfunction has also been identified in CF as a result of the cellular and molecular changes caused by the disease $(5,9,22)$. Alterations of the structure in both ventricles have been reported in 20-47\% of the cases in postmortem studies $(5,6)$. Even though CFTR-related cardiac effects are not entirely elucidated, a commonly proposed 
hypothesis is a disturbance of regulatory functions over calcium channel-related resting membrane potentials and action potentials (9).

Conventional transthoracic echocardiography (TTE) and Doppler echocardiography performed as standard assessments of cardiac function may help identify cardiac involvement in patients with CF. These examinations are, however, often insufficient to demonstrate the presence of early-stage, subclinical cardiac dysfunction. Tissue Doppler echocardiographic imaging (TDI) and two-dimensional speckle tracking echocardiography (STE) are helpful in detecting the presence of subclinical cardiac dysfunction in the early stages of CF $(10,11,12)$.

The objective of this study was to investigate the possibility of a superiority of TDI and STE over standard echocardiography techniques for studying cardiac function in CF patients.

\section{Methods}

\section{Study Subjects}

Local Ethical Committee approval was obtained prior to this prospective study (2014/07-07). A total of 34 patients (17 of each gender, mean age $9.9 \pm 4.9$ years) who were being followed at the Dokuz Eylül University Medical School Allergy Outpatient Clinics were included in the study. Patients eligible for the study group had a positive sweat test and/or gene mutation assay and a stable clinical course with no concomitant cardiac disease. Exclusion criteria included having experienced an acute lower respiratory tract infection in the last three months, being on a waiting list for lung transplantation, and poor quality of the echocardiographic image.

The selected control group consisted of 37 healthy subjects with a comparable age and sex distribution (18 girls and 19 boys with a mean age of $9.8 \pm 4.3$ ) and normal physical, laboratory, electrocardiography (ECG) and TTE results.

\section{Echocardiographic studies}

Echocardiography examinations were performed with an ultrasound device (model iE33, Philips Medical Systems, Netherlands) with an S5-1 MHz transducer by the same observer, who had been blinded to the clinical condition of the patients. Two researchers who were similarly blinded as to the clinical condition and grouping of the patients performed the data analysis off-line.

Two-dimensional echocardiographic images were obtained in standard parasternal, apical and subcostal views. The following end-diastolic and end-systolic parameters were measured in M-mode echocardiography in a parasternal long-axis view: interventricular septal thickness (IVSd and IVSs, respectively), LV dimensions (LVDd and LVDs), and LV posterior wall thickness (LVPWd and LVPWs). LV systolic function was assessed from an apical four-chamber view by measuring the left ventricular ejection fraction (LVEF) using the modified monoplane Simpson's rule. Mitral valve inflow Doppler flow was measured in an apical four-chamber image, E-wave (early filling), A-wave (late filling) and the E/A ratio were derived to evaluate LV diastolic function. Pulmonary artery systolic pressure (PAPs) was determined using the Bernoulli equation on the Doppler continuous wave measurement of tricuspid valve insufficiency flow. Right atrial pressure was evaluated using the inferior vena cava inspiratory collapsibility.

Color TDI images were obtained in the apical view. At least four consecutive cardiac cycles were recorded for each parameter. A Doppler frame scanning rate of 100-140 Hz with $40-80$ frames/sec was used. Early diastolic myocardial wave (e'), atrial diastolic myocardial wave (a'), systolic myocardial wave (s'), total systolic time (TST), ejection time (ET), isovolumetric relaxation time (IVRT) and isovolumetric contraction time (IVCT) were measured during the baseline pulse-wave color TDI examination of the LV free wall and 
of the interventricular septum. The myocardial performance index (MPI) was calculated using the following formula: MPI=IVCT $+\mathrm{IVRT} / \mathrm{ET}$. Diastolic function was assessed by calculating the $\mathrm{E} / \mathrm{e}$ ' ratio.

For the STE evaluation with simultaneous ECG at frame rates of 70-100 frames/s, apical four-chamber (A4C), apical three-chamber (A3C) and apical two-chamber (A2C) images were recorded in the apical view and in the parasternal short axis view the parasternal apical (SAXA), parasternal medial (SAXM) and parasternal basal (SAXB) images. At least four consecutive cardiac cycles were recorded for each parameter. The recorded images were transferred onto DVD and analyzed on a computer using the QLAP software (Philips Medical Systems). The mitral annulus lateral and septal and the LV apical endocardial planes were marked for each three apical chambers, providing for automated generation of the LV wall by the program. The LV endocardial-myocardial border was adjusted manually on the systolic frames. Peak systolic strain, peak systolic strain rate and global strain values were calculated automatically by the software for six segments (apical, middle, basal) based on septum and LV lateral wall motion values. In all three parasternal views, the endocardial-myocardial border was adjusted manually after the program had automatically generated the ventricular wall. Patients whose results were abnormal due to poor image quality were excluded from the study.

\section{Statistical Analysis}

Statistical analyses were performed using the Statistical Package for the Social Sciences (SPSS) software, version 22.0 (SPSS Inc.; Chicago, IL, USA). Continuous data were described as mean \pm standard deviation while categorical data were presented as numbers of patients. The Chi-squared test was used for comparison of categorical variables, while parametric continuous variables were compared by Student's t-test. Data were checked for normal distribution by the Kolmogorov-Smirnov test. A value of $\mathrm{p}<0.05$ was considered statistically significant. Evaluations were repeated by a second, independent observer, to assess interobserver variability. Interobserver variability was calculated as the absolute difference divided by the average of the two observations for all parameters.

\section{Results}

\section{Cohort characteristics}

The 34 patients with a diagnosis of CF included in the study were 17 girls and 17 boys with a mean age of $9.94 \pm 4.97$ years, while of the 37 healthy volunteers, whose mean age was $9.85 \pm 4.33$; 18 were female and 19 male. No significant differences between the two groups were detected with regard to sex, age or weight $(\mathrm{p}>0.05)$. Testing for gene mutation was positive in $19(55.8 \%)$ and the sweat test was positive in 30 $(88.2 \%)$, with a mean score of $96.70 \pm 24.63$ for the latter. Gastrointestinal and pulmonary involvement was present in $30(88.2 \%)$ and $23(94.1 \%)$ patients, respectively. In $16(64 \%)$ of the 25 patients with a pulmonary involvement determined on the CT-scan, bronchiectasis was present. Of the CF patients, $4(11.7 \%)$ had a history of more than 10 in-patient hospital admissions for pulmonary infection; the total number of such admissions was 5-10 in $10(29.4 \%)$ and was less than 5 in 15 (44.1\%) patients. Colonization by Pseudomonas aeruginosa was detected in $13(38.2 \%)$ and that by Staphylococcus aureus in $11(32.3 \%)$. Of 19 patients who were subjected to respiratory function testing, 13 (68.4\%) showed an abnormal result; the remaining 6 $(31.6 \%)$ were unremarkable in this regard.

\section{Echocardiographic Findings}

M-mode and Doppler echocardiography results are summarized in Table 1. While no difference could be detected between the patients and volunteer groups with regard to LV measurements IVSd, IVSs, LVPWd, LVPWs, EF and FS, there as a significant difference between them in LVDd and LVDs measurements. Pulmonary artery systolic pressure (PAPs) was significantly higher in the CF group compared to the controls 
$(32.50 \pm 4.09$ and $28.96 \pm 2.82 \mathrm{mmHg}$, respectively; $\mathrm{p}<0.001)$. Diastolic pulmonary artery pressure (PAPd) was $10.57 \pm 3.68 \mathrm{mmHg}$ in the patients and $9.00 \pm 2.08 \mathrm{mmHg}$ in the control patients, with no statistically significant difference detected. Similarly, no statistically significant difference could be evidenced between the two groups in the Doppler echocardiography parameters E-wave, A-wave, E/A ratio and E/e' ratio.

Color TDI results are summarized in Table 2. Color TDI measurement of LV parameters also evidenced some systolic and diastolic dysfunction. When compared to the controls by TDI, CF patients had shorter TST $(343.7 \pm 37.5 \mathrm{~ms}$ vs. $367.8 \pm 38.6 \mathrm{~ms}$ respectively, $\mathrm{p}=0.009)$, $\mathrm{EF}(260.7 \pm 32.8 \mathrm{~ms}$ vs. $288 \pm 38.7 \mathrm{~ms}, \mathrm{p}=0.002)$ and increased MPI $(0.32 \pm 0.04$ vs. $0.28 \pm 0.05, \mathrm{p}=0.001)$. Interventricular septal TST was reduced in CF patients $(341.1 \pm 38.6 \mathrm{~ms}$ vs. $361.1 \pm 3.2 \mathrm{~ms}$ in controls, $\mathrm{p}=0.027)$, ET was relatively shortened $(256.9 \pm 35$ ms vs. $282.2 \pm 33.2 \mathrm{~ms}, \mathrm{p}=0.002)$, IVRT lengthened $(47.6 \pm 5.8 \mathrm{~ms}$ vs. $44.4 \pm 5.3 \mathrm{~ms}, \mathrm{p}=0.017)$ and MPI was increased ( $0.33 \pm 0.04$ vs. $0.28 \pm 0.03, \mathrm{p}=0.000)$.

\section{Speckle Tracking Echocardiography}

LV longitudinal myocardial strain measurements indicated the following reductions in segmental circumferential myocardial strain compared to the control group: apical septal $(-20.0 \pm 4.8$ vs. $-23.4 \pm 5.8, \mathrm{p}=0.015)$, apex (-20.0 \pm 4.8 vs. $-23.4 \pm 5.8, \mathrm{p}=0.003)$, AC3 chamber mid inferolateral $(-18.7 \pm 4.1$ vs. $-22.2 \pm 5.6, \mathrm{p}=0.004)$, basal anteroseptal ( $-17.8 \pm 3.9$ vs. $-21.4 \pm 5.7, \mathrm{p}=0.004)$, mid inferior $(-18.7 \pm 4.1$ vs. $-21.5 \pm 6.2, \mathrm{p}=0.044)$, apical inferior $(-19.9 \pm 4.3$ vs. $-22.2 \pm 4.4, \mathrm{p}=0.027)$, mid anterior $(-18.8 \pm 3.9$ vs. $-21.7 \pm 5.9, \mathrm{p}=0.025)$. Global strain and total global strain values did not appear to be different (Table 3). Circumferential myocardial strain measurements of the LV wall showed significant reductions in CF patients compared to controls in the basal inferior $(-17.9 \pm 7.2$ vs. $-21.2 \pm 6, \mathrm{p}=0.049)$, mid inferior $(-17.8 \pm 7$ vs. $-23.9 \pm 6.6, \mathrm{p}=0.001)$ and apical anterior $(-21.6 \pm 6.6$ vs. $-27.5 \pm 5.2, \mathrm{p}<0.001)$ segments. No difference was observed between global strain and total global strain values (Table 4). Longitudinal strain rate measurements of $\mathrm{CF}$ patients by segment, compared to the controls by segment, were as follows: in $\mathrm{A} 4 \mathrm{C}$ mid anterolateral $(-1.5 \pm 0.3$ vs. $-2.0 \pm 0.5, \mathrm{p}=0.001)$, in $\mathrm{A} 3 \mathrm{C}$ basal inferolateral ( $-1.6 \pm 0.3$ vs. $-1.9 \pm 0.6, \mathrm{p}=0.030)$, in $\mathrm{A} 2 \mathrm{C}$ mid inferior $(-1.7 \pm 0.4$ vs. $-2.1 \pm 0.7$, $\mathrm{p}=0.010)$, apex $(-1.3 \pm 0.2$ vs. $-1.5 \pm 0.5, \mathrm{p}=0.023)$, apical anterior $(-1.4 \pm 0.3$ vs. $-1.7 \pm 0.6, \mathrm{p}=0.041)$. Circumferential strain rate measurements were also lower in CF patients than in healthy controls in the following parameters: in basal view, in the basal anteroseptal ( $-2.0 \pm 0.4$ vs. $-2.4 \pm 0.5, \mathrm{p}=0.003)$ and basal anterolateral $(-1.6 \pm 0.4$ vs. $-2.0 \pm 0.7, \mathrm{p}=0.040)$; in the medial view, in the mid anterior $(-1.7 \pm 0.5$ vs. $-2.0 \pm 0.5, \mathrm{p}=0.039)$, mid inferolateral $(-1.7 \pm 0.4$ vs. $-2.0 \pm 0.6, \mathrm{p}=0.035)$ and mid inferoseptal segments $(-1.7 \pm 0.4$ vs. $-1.9 \pm 0.3$, $\mathrm{p}=0.012)$ (Tables 3,4$)$.

\section{Discussion}

$\mathrm{CF}$ is known to affect cardiac function. Systolic and diastolic LV dysfunction in CF patients has been well characterized. TTE evaluation in M-mode and Doppler echocardiography generally shows normal results in the early stages of cardiac involvement. This cardiac dysfunction in its subclinical stage may be characterized using methods such as TDI and STE. While TDI provides global parameters of ventricular function, STE may be used to selectively evaluate areal ventricular function. STE thus provides superior evaluation quality compared to TDI $(10,11,12)$.

The baseline assessment in our study used physical examination, ECG and TTE. Systolic and diastolic LV functions were evaluated by TTE, Doppler, TDI and STE. While ventricular function as assessed by TTE appeared to be normal in all cases, pathologic findings of systolic and diastolic function were revealed by TDI and STE. Three recently published reports evaluate ventricular function in CF patients using STE. Two of these studied LV and RV function in adults; the third reports RV function in children as measured by TDI, strain and strain rate. All three studies compared TDI to STE. While ventricular function appeared to be normal in TTE, both techniques gave results confirming subclinical ventricular dysfunction $(5,8,10)$. 
PAPs as measured based on the tricuspit regurgitation (TR) jet was higher in the CF patients group than in controls. In their study of 35 adult CF patients with a mean age of 27.14 years, Thomas et al.found similarly high PAPs values (1). In this study, 13 patients diagnosed with pulmonary hypertension were found to have a PAPs higher than $35 \mathrm{mmHg}$. In our study, 6 of the CF patients had a PAPs value exceeding $35 \mathrm{mmHg}$. Özçelik et al. found the PAPs of pediatric CF patients $(\mathrm{n}=18$, mean age 7.7) to be normal (8). LV compression by RV pressure elevation and an abnormal interventricular septal motion may also affect LV function (5).

In their report of an evaluation by TDI of the LV function of 8 patients with an average age of 35, Sellers et al . find a higher IVRT in CF patients compared to controls (10). While S-wave velocities may appear to be reduced in the patient group relative to the controls, no statistical significance was detected. The IVRT increase and S-wave velocity reduction in our CF patients was similarly not confirmed as statistically significant. TDI studies in CF patients have often been conducted in adult populations. The studies focused mainly on the IVRT increase and reduction in S-wave velocities. Özçelik et al., who also evaluated LV function by TDI, could not detect a statistically significant difference between CF patients and controls with regard to E/A and E/e' ratios and the MPI. While our results were similar for E/A and E/e', a clear difference between patients and control subjects was evidenced for MPI, which was higher in the CF patients. MPI is a global indicator of LV function. It increases with the development of LV dysfunction. We interpreted it as an indicator of LV dysfunction.

STE is a new technique for evaluating ventricular function. The use of strain and strain rate parameters to evaluate regional deformation is an alternative to conventional echocardiography. Strain here expresses the percent dimensional deformation occurring in the object, while strain rate is the velocity of such deformation. Published reports have shown the superiority of these parameters over TTE and TDI in identifying ventricular dysfunction. This technique also seems superior to others in evaluating local ventricular function in addition to the global one $(15,16,17)$.

In 41 patients with a mean age of 24, Labombarda et al. used strain and strain rate to evaluate LV free wall and septal function (5). They found free wall strain and strain rate and septal strain values to be significantly lower in patients compared to control subjects. While also appearing lower, the septal strain rate values were not significantly different than those of the controls. Sellers et al. compared peak systolic strain and strain rates in LV STE studies of adult CF patients to reference values and found them to be lower (10). We did a similar evaluation of LV strain and strain rate. While the two published STE reports evaluated ventricular function globally, our study was different in that it additionally measured local LV function. Of longitudinal myocardial strain measurements, APS and apex segments in A4C view, MIL and BAS in A3C, MI, API and $\mathrm{MA}$ in $\mathrm{A} 2 \mathrm{C}$ were significantly reduced compared to controls. As for circumferential myocardial strain measurements, the API segment in the apical, the MI segment in the medial and the BI segment in the basal view were also significantly lower than in control subjects. While global and total global strain values appeared to be lower in 6 windows, this difference was not confirmed by a detected statistical significance. A statistically significant reduction was established in CF patients in five segments (MAL, BIL, MI, apex, APA) in the longitudinal, and five (BAS, BAL, MA, MIL, MIS) in the circumferential strain rate measurements.

LV dysfunction detected by TDI and STE in CF patients is attributed to different causes, primarily chronic hypoxia, chronic inflammation, myocardial fibrosis and RV dysfunction $(5,18,19)$. LV compression by a dilated RV and interventricular septal motion abnormalities may also be counted as additional causes of this LV dysfunction. Elevated plasma angiotensin II and aldosterone levels may also be observed in CF patients. As a result, myositis with fibroblastic cell proliferation and an increased protein synthesis leading directly to myocardial fibrosis may develop. Angiotensin-converting enzyme inhibitors and angiotensin II receptor antagonists may thus be included in treatment plans $(5,18,19)$. The CFTR has been characterized in myositis; it has a regulatory function on resting potentials, calcium-mediated depolarization and beta-adrenergic stimulation. Disruption of regulation mechanisms at the cellular level due to CFTR gene defects creates a favorable background for cardiac dysfunction $(9,22)$. Hyperglycemia, which develops in $32 \%$ of CF patients over age 25 as a result of pancreatic beta-cell damage, may lead to myocardial function impairment through an increase in myocardial stiffness and impaired contractility caused by the increased protein glycosylation 
$(20,21)$.

As CF patients benefit from an increased life expectancy thanks to effective treatment and lung transplantation, problems of myocardial dysfunction may be expected to be increase in frequency. Myocardial dysfunction in its subclinical stage may be characterized using methods such as TDI and STE. The ease of use of both these techniques and the availability of reference values may suggest their possible use in the follow-up of $\mathrm{CF}$ patients. Further studies are needed to determine whether these findings have clinical significance.

\section{Study Limitations}

Our study has several limitations. The first was the impossibility of using the STE software available to us to evaluate the three-dimensional structure of RV geometry, while RV dysfunction is the main focus in CF. The second was that the number of subjects was small. Finally, invasive hemodynamic data, exercise testing and cardiac MRI were not available at the same time for correlation with the echocardiographic measurements.

Contributors TD,SI is the guarantor of this work and, as such, had full access to all the data in the study and takes responsibility for the integrity of the data and the accuracy of the data analysis. OK wrote the manuscript and researched data. NU, NY and NU reviewed/edited the manuscript. OK and MK performed the echocardiographic evaluation.

\section{REFERENCES}

1. Bright-Thomas RJ, Ray SG, Webb AK. Pulmonary artery pressure in cystic fibrosis adults: Characteristics, clinical correlates and long-term follow-up. Journal of Cystic Fibrosis. 2012;11(6):532-38.

2. Brıght-Tomas RJ, Webb AK. The heart in cystic fibrosis. J R Soc Med. 2002;95(41):2-10.

3. Tonelli AR, Fernandez-Bussy S, Lodhi S, et al. Prevalence of pulmonary hypertension in endstage cystic fibrosis and correlation with survival. The Journal of Heart and Lung Transplantation. 2010;29(8):865-72.

4. Bano-Rodrigo A, Salcedo-Posadas A, Villa-Asensi JR, et al. E. Right ventricular dysfunction in adolescents with mild cystic fibrosis. Journal of Cystic Fibrosis. 2012;11(4):274-80.

5. Labombarda F, Pellissier A, Ellafi M, et al. Myocardial Strain Assessment in Cystic Fibrosis. Journal of the American Society of Echocardiography. 2011;24(9):1037-45.

6. Koelling TM, Dec GW, Ginns LC, et al. Left ventricular diastolic function in patients with advanced cystic fibrosis. Chest. 2003;123:1488-94.

7. Rovedder PME, Ziegler B, Pinotti AFF, et al. Prevalence of pulmonary hypertension evaluated by Doppler echocardiography in a population of adolescent and adult patients with cystic fibrosis. J. Bras Pneumol. 2008;34(2):83-90.

8. Ozcelik N, Shell R, Holtzlander M, et al. Decreased Right Ventricular Function in Healthy Pediatric Cystic Fibrosis Patients Versus Non-Cystic Fibrosis Patients. Pediatric Cardiology. 2013;34(1):159-64.

9. Sellers ZM, Kovacs A, Weinheimer CJ, et al. Left ventricular and aortic dysfunction in cystic fibrosis mice. Journal of Cystic Fibrosis. 2013;12(5):517-24.

10. Sellers ZM, McGlocklin L, Brasch A. Strain rate echocardiography uncovers subclinical left ventricular dysfunction in cystic fibrosis. Journal of Cystic Fibrosis. doi:10.1016/j.jcf.2015.03.010.

11. Ionescu AA, Ionescu AA, Payne N, et al. Subclinical right ventricular dysfunction in cystic fibrosis. American Journal of Respiratory and Critical Care Medicine. 2001;163(5):1212-18.

12. Matsui H, Satomi G, Yasukochi S, et al. Evaluation of right ventricular contraction by myocardial strain in children using a two-dimensional tissue tracking method. Pediatr Cardiol. 2008;29:377-81.

13. Lopez-Candales A, Rajagopalan N, Gulyasy B, et al. Differential strain and velocity generation along the right ventricular free wall in pulmonary hypertension. Can J Cardiol. 2009;25:73-77.

14. Simon MA, Rajagopalan N, Mathier MA, et al. Tissue Doppler imaging of right ventricular decompensation in pulmonary hypertension. Congest Heart Fail. 2009;15:271-76.

15. Takigiku K, Takeuchi M, Izumi C, et al. Normal range of left ventricular 2-dimensional strain. Circ J. 2012;76(11):2623-32. 
16. Dandel M, Lehmkuhl H, Knosalla C, et al. Strain and Strain Rate Imaging by Echocardiography Basic Concepts and Clinical Applicability. Curr Cardiol Rev. 2009;5(2):133-148.

17. Geyer H, Caracciolo G, Abe H, et al. Assessment of myocardial mechanics using speckle tracking echocardiography: fundamentals and clinical applications. J Am Soc Echocardiogr. 2010;23(4):351-69.

18. Gonzales MP, Suarez L, Camarero C, et al. Myocardial fibrosis in 2 children with cystic fibrosis. An Esp Pediatr. 1987;27:382-4.

19. Wiebicke W, Artlich A, Gerling I. Myocardial fibrosis: a rare complication in patients with cystic fibrosis. Eur J Pediatr. 1993;152:694-6.

20. Bismuth E, Laborde K, Taupin P, et al. Glucose tolerance and insulin secretion, morbidity, and death in patients with cystic fibrosis. J Pediatr 2008;152:540-5.

21. Fang ZY, Prins JB, Marwick TH. Diabetic cardiomyopathy: evidence, mechanisms, and therapeutic implications. Endocr Rev 2004;25:543-67.

22. Kuzumoto M, Takeuchi A, Nakai H, et al. Simulation analysis of intracellular $\mathrm{Na}^{+}$and $\mathrm{Cl}^{-}$homeostasis during beta 1-adrenergic stimulation of cardiac myocyte. Prog Biophys Mol Biol. 2008;96:171-86. 